\title{
Design of Functions and Hardware Structure of Monitoring System of Ventilators
}

\author{
Guoli Sun ${ }^{1, a}$ \\ ${ }^{1}$ Jiangsu Province Xuzhou Technician Institute, Xuzhou, 221000, China \\ ${ }^{a}$ email
}

Keywords: Ventilators, Monitoring, Structure design

\begin{abstract}
Monitoring system of the ventilator is one of the fast developing technologies in the automation and informationization process of coal mine. At present, all the main fans of the mine are equipped with the on-line monitoring system. It is responsible for monitoring and measuring the parameters and indicators of the fan and describing the system operation situation of the controlled object, providing accurate data reference for the safety production, fault diagnosis and efficiency analysis. According to the scale and characteristics of the controlled system, the correct selection and construction of the control system is an important part of the design of the ventilator monitoring system.
\end{abstract}

\section{Several Kinds of Control System in Industrial Applications}

Relay control system is widely used in various fields of industrial production, which is mainly composed of four parts: input circuit, control circuit, output circuit and production site. The input circuit is composed of a button switch, a position switch, a sensor, etc., which is used to send the control signal to the system. The output circuit is composed of a contractor, an electromagnetic valve, etc., which can be used to control various controlled objects, such as electric motors, electric furnaces, valves. The control circuit is the core of the control system, which connects the individual relays and electronic components through the wire. Production site refers to the controlled object or production process.

Relay control system has the following characteristics: the system is composed of many relays; the realization of the control function is completed by wiring. The control speed is low, and the control is realized by mechanical action. More contacts, more connections, high failure rate, poor scalability. The relay control system has played an irreplaceable role in industrial production, with the progress of technology, gradually expand the scale of production, the increasingly fierce market competition, the relay control system is more and more difficult to adapt, because the relay control circuit is usually for a fixed design action sequence or the production process, the control function is relatively simple once the action sequence changes, or changes in the production process, the system will re design, wiring, assembling and debugging, obviously cannot meet the requirements of increasingly complex control.

At present, the development direction of relay manufacturing technology is diverse, small, solid, combination, development of relay control system is still relatively slow. It cannot keep up with the growing industrial control system of high precision control requirements. Therefore, to force people to give up the original relay control system has been dominant, the new control system to replace the relay control system can be developed, the relay control system status gradually by other more advanced control system instead of.

Fieldbus control system is a kind of digital, bidirectional transmission and multi branch communication network and monitoring system, which is connected with the intelligent equipment and automation system on site. FCS went to practical applications in 1990s in the last century, which is rapidly developing today. It is the world's latest control system. Fieldbus control system is a hot spot in automation technology, and it is being paid more and more attention by domestic and international automation equipment manufacturers and users. Fieldbus control is mainly composed of 
the following categories: transmitter, actuator, converter, interface equipment. Commonly used PLC includes CPU module, I/O module, memory, power module, backplane or rack.

\section{Main Functions of Online Monitoring System}

Fan monitoring system developed to the fan in the process of running to the class parameters on-line real-time acquisition, processing and controlling, to grasp the running state of the fan on the whole, and the abnormal situation and fault processing is realized according to the coal enterprise management procedures and the need for the professional automation system the automatic control of main fan through the host computer monitoring system needs to achieve the main performance parameters, to achieve the set automatic control program, the main task of realizing mine automation network connection and data upload.

Main Monitoring Functions of Monitoring System. The monitoring contents include the operation parameters of the fan, the performance parameters of the motor, the switch state, the fault diagnosis signal, the line sound and the color alarm. The main features are described as follows. The real time data value of static pressure and total pressure of the fan inlet is measured, which is reflected in the form of real-time parameters and real-time curves. The real time data value of the fan flow is reflected in the form of real-time parameters and real time curves. The monitoring of important equipment of fan bearings, motor bearings, such as motor stator temperature, to reflect the real-time parameters, dynamic curve, analog bar graph form, at the same time recording, data storage, historical curve, report generation. The axial and radial vibration of the front and rear bearings of the fan are monitored in the form of real-time parameters and real time curves. The voltage, current and power of the fan are monitored in real time and in the form of real time curve. Monitor the operation state of the motor, the positive and negative state, the state of the fault, the open and close state of the air door, the standby of the frequency converter, the opening and stopping, the light fault, the heavy fault state, and the indicating lamp can be visually displayed. To display the whole ventilation system of the operating state of intuitive, animation simulation system of running state for visual observation, fan motor, inverter, change trend and main parameters. The relationship between the main performance parameters of negative pressure and flow rate of fan with time to display the form of dynamic curve, easy to observe the parameters change with time, while the parameter value is displayed in a text box. The system stores the main parameters of the fan, and the time interval is every minute, every hour, and every day. Generate the corresponding report on all the important parameters, including the maximum, minimum, average and standard variance statistics in the corresponding time period, which is convenient for the operators and managers to inquire and print. The main parameters of the fan performance, such as the negative pressure of the fan, the fan flow rate, the power of the motor, the fan efficiency and so on, are generated. The establishment of the main parameters and equipment fault alarm mechanism of fan bearing temperature, bearing vibration, temperature of motor bearing, motor stator temperature setting limits, to limit alarm; alarm equipment failure of fan and motor. Limit and fault parameters according to user settings, alarm by sound, and can record and print alarm information. In order to prevent unauthorized users of fan equipment operation authorized by the personnel on duty or because of disoperation view data such as routine operation, the system set up different operating authority, respectively according to different levels of users, increase the science of management, accident or loss caused by accidental operation. In the system set up online help system, including the system operation steps, common faults and processing methods, safety rules.

Main Control Functions. The main control functions of the system are as follows. Complete fan normal start and stop, switch the fan control; and according to the monitoring signal, understand the running state of the fan, when the main fan fault occurs, the judgment result output corresponding control signal by the computer, stop the main fan, and start the standby motor. By starting, stopping and adjusting frequency operation of the frequency converter, the motor can be started smoothly and gently stopped, and the fluctuation of the power grid caused by the large-scale equipment can be reduced, and the motor and the fan equipment can be protected. At the same time, according to the 
actual power needed to adjust the frequency of power supply through the frequency converter, to achieve the effect of wind on demand, to achieve energy saving operation. The opening and closing of the damper and the control of other relevant control system.

\section{Determination of System Design Scheme}

Basic Requirements of System Design. As an industrial control system, usually for different control requirements for system design, but the overall design requirements of the control system, the basic requirements of various systems are generally the same. Good operating performance. The system hardware and software need to consider the use and maintenance of good operability, simple, easy to understand, easy to learn and master, the operation order as simple as possible. Reliability is one of the most important requirements of the system design. In the design of the system, the high performance industrial control computer should be selected. The control scheme and the software design should be reliable. Good versatility, easy to expand. A control device based on microcomputer, can control multiple devices and process parameters, considering all kinds of different equipment and suitable in a certain range of different objects in the design, the control device does not need to be changed quickly to adapt to the new situation. In order to realize the real-time monitoring and control of the industrial production process, it is to realize the real-time monitoring of the production. Design cycle to meet the requirements of the production schedule, system selection of equipment to meet the monitoring requirements of the premise, the economy is good, cost-effective.

Determination of System Scheme. Ventilator monitoring system is a part of the whole monitoring. At the same time, it is a complete and independent system, overall, monitoring parameters of main fan for a total of seven types of signals, fifty total measuring points, twenty control points, and mine ring network connection communication, belonging to the medium control system. Comprehensive control requirements of mine ventilator work environment, users of the control system and the current mainstream products and other factors, this paper decided to use PLC as the basic hardware, software configuration software as the core, the allocation of various types of equipment, construction monitoring and control system. Specifically, the analog input module of the programmable controller (AI module) and switch input module (DI module) through real-time data acquisition, digital output module (AO module) and the switch output module (DO module) output, through the communication module of PLC data transmission to the monitoring station computer and Ethernet communication. After the calculation, comparison, tabulation, printing, alarm, query and other functions through the host computer.

After the completion of the hardware selection, it is necessary to configure the STEP program, the hardware will be arranged according to the physical order in the configuration window of the software. In the configuration window, the system will automatically assign a unique address to each configuration of the module, some models of CPU can also be based on user needs to modify the configuration of the module address. The properties of the S7 programmable controller and the module can be preset to the default value, so that in many cases, it is not necessary to configure it. In the following cases, the configuration is required: if you want to change the default parameters of the module (for example, enable module hardware interrupts). If you want to configure the communication connection. For stations with distributed I/O (PROFIBUS DP or PROFINET IO). For S7-400 stations with multiple CPU (multi value calculations) or extended racks. Fault tolerant $(\mathrm{H})$ programmable control system. 


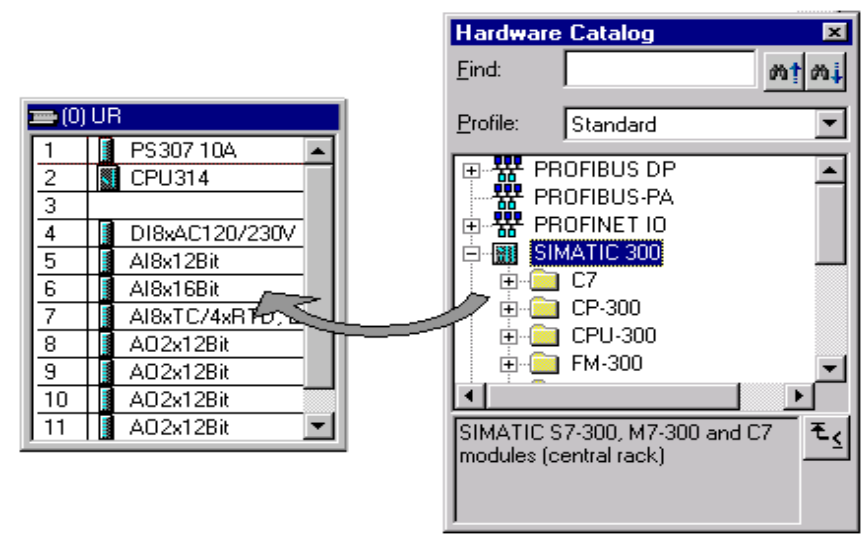

Fig. 1: Operation Process of Hardware Configuration

The basic steps are as follows: the hardware configuration for the configuration of the configuration window, the programmable controller will use two windows: one for the station frame structure placed station window; two is "the hardware directory window, hardware components required to choose from, such as frame, module and interface module. In the hardware directory, select a hardware component; drag and drop to copy the selected component into the destination window. In the construction of innovative activities in the green mine, coal mine main fan as a large mining equipment, bear the important role of transporting fresh air to the underground, dilution of harmful gases". The safe and efficient operation is related to the safety of mine production. Through the use of modern monitoring methods, the fan in the operation of the various parameters of real-time monitoring and control, from the macro to assess the overall operation of the state of the fan. It has important practical significance to improve the management level of ventilation equipment and ensure the safe and efficient operation of mine.

\section{References}

[1] Wu Guihui, China's Energy Situation and Energy Development Measures [J]. Energy and Energy Conservation, 2011(1): 10-11.

[2] Zhang Ping, Liu Yanhu, The Background Analysis of Building Saving Society and Important Territory [J]. China Chemical Industry Equipment, 2006(3): 15-18.

[3] Chen Qingru, Wang Haifeng, Clean Processing and Utilization of Coal Energy[J]. The Chinese Journal of Process Engineering, 2006(6): 35-38.

[4] Dongke Zhang, Energy options in sustainable development[J]. Journal of Fuel Chemistry and Technology, 2005(8): 399-406. 